\title{
MAGIC observations of MWC 656, the only known Be/BH system (Research Note)
}

J. Aleksić ${ }^{1}$, S. Ansoldi ${ }^{2}$, L. A. Antonelli ${ }^{3}$, P. Antoranz ${ }^{4}$, A. Babic ${ }^{5}$, P. Bangale ${ }^{6}$, J. A. Barrio ${ }^{7}$, J. Becerra González $^{8,25}$, W. Bednarek ${ }^{9}$, E. Bernardini ${ }^{10}$, B. Biasuzzi ${ }^{2}$, A. Biland ${ }^{11}$, O. Blanch ${ }^{1}$, S. Bonnefoy ${ }^{7}$, G. Bonnoli ${ }^{3}$, F. Borracci ${ }^{6}$, T. Bretz ${ }^{12,26}$, E. Carmona ${ }^{13}$, A. Carosi ${ }^{3}$, P. Colin ${ }^{6}$, E. Colombo ${ }^{8}$, J. L. Contreras ${ }^{7}$, J. Cortina ${ }^{1}$, S. Covino ${ }^{3}$, P. Da Vela ${ }^{4}$, F. Dazzi ${ }^{6}$, A. De Angelis ${ }^{2}$, G. De Caneva ${ }^{10}$, B. De Lotto ${ }^{2}$, E. de Oña Wilhelmi ${ }^{14}$, C. Delgado Mendez ${ }^{13}$, D. Dominis Prester ${ }^{5}$, D. Dorner ${ }^{12}$, M. Doro ${ }^{15}$, S. Einecke ${ }^{16}$, D. Eisenacher ${ }^{12}$, D. Elsaesser ${ }^{12}$, D. Fidalgo ${ }^{7}$ M. V. Fonseca ${ }^{7}$, L. Font ${ }^{17}$, K. Frantzen ${ }^{16}$, C. Fruck ${ }^{6}$, D. Galindo ${ }^{18}$, R. J. García López ${ }^{8}$, M. Garczarczyk ${ }^{10}$, D. Garrido Terrats ${ }^{17}$, M. Gaug ${ }^{17}$, N. Godinovic ${ }^{5}$, A. González Muñoz ${ }^{1}$, S. R. Gozzini ${ }^{10}$, D. Hadasch ${ }^{14,27}$,

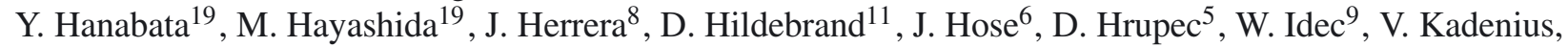
H. Kellermann ${ }^{6}$, M. L. Knoetig ${ }^{11}$, K. Kodani ${ }^{19}$, Y. Konno ${ }^{19}$, J. Krause ${ }^{6}$, H. Kubo ${ }^{19}$, J. Kushida ${ }^{19}$, A. La Barbera ${ }^{3}$, D. Lelas ${ }^{5}$, N. Lewandowska ${ }^{12}$, E. Lindfors ${ }^{20,28}$, S. Lombardi ${ }^{3}$, F. Longo ${ }^{2}$, M. López ${ }^{7}$, R. López-Coto ${ }^{1}$, A. López-Oramas ${ }^{1, \star}$, E. Lorenz ${ }^{6}$, I. Lozano ${ }^{7}$, M. Makariev ${ }^{21}$, K. Mallot ${ }^{10}$, G. Maneva ${ }^{21}$, N. Mankuzhiyil ${ }^{2,29}$, K. Mannheim ${ }^{12}$, L. Maraschi ${ }^{3}$, B. Marcote ${ }^{18}$, M. Mariotti ${ }^{15}$, M. Martínez ${ }^{1}$, D. Mazin ${ }^{6}$, U. Menzel ${ }^{6}$, J. M. Miranda ${ }^{4}$, R. Mirzoyan ${ }^{6}$, A. Moralejo ${ }^{1}$, P. Munar-Adrover ${ }^{18, \star}$, D. Nakajima ${ }^{19}$, V. Neustroev ${ }^{20}$, A. Niedzwiecki ${ }^{9}$, K. Nilsson ${ }^{20,28}$, K. Nishijima ${ }^{19}$, K. Noda ${ }^{6}$, R. Orito ${ }^{19}$, A. Overkemping ${ }^{16}$, S. Paiano ${ }^{15}$, M. Palatiello ${ }^{2}$, D. Paneque ${ }^{6}$, R. Paoletti ${ }^{4}$, J. M. Paredes ${ }^{18}$, X. Paredes-Fortuny ${ }^{18}$, M. Persic ${ }^{2,30}$, J. Poutanen ${ }^{20}$, P. G. Prada Moroni ${ }^{22}$, E. Prandini ${ }^{11}$, I. Puljak ${ }^{5}$, R. Reinthal ${ }^{20}$, W. Rhode ${ }^{16}$, M. Ribó ${ }^{18}$, J. Rico ${ }^{1}$, J. Rodriguez Garcia ${ }^{6}$, S. Rügamer ${ }^{12}$, T. Saito ${ }^{19}$, K. Saito ${ }^{19}$, K. Satalecka ${ }^{7}$, V. Scalzotto ${ }^{15}$, V. Scapin ${ }^{7}$, C. Schultz ${ }^{15}$, T. Schweizer ${ }^{6}$, A. Sillanpää20 ${ }^{20}$ J. Sitarek ${ }^{1}$, I. Snidaric ${ }^{5}$, D. Sobczynska ${ }^{9}$, F. Spanier ${ }^{12}$, A. Stamerra ${ }^{3}$, T. Steinbring ${ }^{12}$, J. Storz ${ }^{12}$, M. Strzys ${ }^{6}$, L. Takalo ${ }^{20}$, H. Takami ${ }^{19}$,

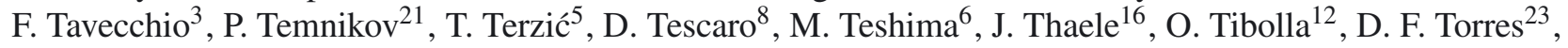
T. Toyama ${ }^{6}$, A. Treves ${ }^{24}$, P. Vogler ${ }^{11}$, M. Will ${ }^{8}$, R. Zanin ${ }^{18}$, (the MAGIC Collaboration), J. Casares ${ }^{8}$, and J. Moldón ${ }^{18,31}$

(Affiliations can be found after the references)

Received 29 August 2014 / Accepted 5 February 2015

\section{ABSTRACT}

Context. MWC 656 has recently been established as the first observationally detected high-mass X-ray binary system containing a Be star and a black hole (BH). The system has been associated with a gamma-ray flaring event detected by the AGILE satellite in July 2010. Aims. Our aim is to evaluate whether the MWC 656 gamma-ray emission extends to very high energy (VHE $>100 \mathrm{GeV}$ ) gamma rays. Methods. We observed MWC 656 with the MAGIC telescopes for $\sim 23 \mathrm{~h}$ during two observation periods: between May and June 2012, and in June 2013. During the last period, observations were performed contemporaneously with X-ray (XMM-Newton) and optical (STELLA) instruments.

Results. We did not detect the MWC 656 binary system at TeV energies with the MAGIC telescopes in either of the two campaigns. Upper limits (ULs) to the integral flux above $300 \mathrm{GeV}$ have been set, as well as differential ULs at a level of $\sim 5 \%$ of the Crab nebula flux. The results obtained from the MAGIC observations do not support persistent emission of VHE gamma rays from this system at a level of 2.4\% the Crab flux.

Key words. astroparticle physics - binaries: general - gamma rays: stars - X-rays: binaries - stars: individual: MWC 656

\section{Introduction}

High-mass X-ray binaries (HMXBs) are systems composed of a massive $\operatorname{star}\left(M_{\star} \geq 10 M_{\odot}\right)$ and a compact object, either a black hole $(\mathrm{BH})$ or a neutron star (NS). The search for $\mathrm{GeV}$ and $\mathrm{TeV}$ emission from HMXBs has been the aim of extensive studies during the past few decades. Despite the large number of observations devoted to the search, only a few of these

* Corresponding author: A. López-Oramas, e-mail: alopez@ifae.es; P. Munar-Adrover, e-mail: pmunar@am.ub.es systems have been confirmed as gamma-ray emitters. A particular group of five systems are regularly detected at TeV energies: the gamma-ray binaries (see Dubus 2013, and references therein). Two other HMXBs have been the object of extensive searches: Cygnus X-3, which emits in the high-energy (HE; $100 \mathrm{MeV}<E<100 \mathrm{GeV}$ ) domain (Fermi-LAT Collaboration et al. 2009; Tavani et al. 2009b), and Cygnus X-1, which has been reported to emit at HE (Sabatini et al. 2013; Malyshev et al. 2013; Bodaghee et al. 2013) and showed a $\sim 4 \sigma$ excess at very high energy (VHE; Albert et al. 2007). To investigate the gamma-ray mechanisms in this type of sources, observational campaigns on other HMXBs have been carried out. The recently 
discovered object MWC 656 (Lucarelli et al. 2010) is an HMXB system and has been proposed as a new gamma-ray binary candidate (Williams et al. 2010).

In July 2010, AGILE (Tavani et al. 2009a) detected a gamma-ray point-like source dubbed AGL J2241+4454 with a significant excess above 5 sigma, displaying an integral flux above $100 \mathrm{MeV}$ of $15 \times 10^{-7} \mathrm{ph} \mathrm{cm}^{-2} \mathrm{~s}^{-1}$ (Lucarelli et al. 2010). The source was first detected during the period between 25 July at 01:00 UT (MJD = 55 402.042) and 26 July 2010 at 23:30 UT $(\mathrm{MJD}=55403.979)$. The source is located at $(l, b)=\left(100.0^{\circ},-12.2^{\circ}\right) \pm 0.6^{\circ}(95 \%$ stat. $) \pm 0.1^{\circ}$ (syst.). At the time of writing, no further flares from this source have been reported and no spectrum has been published.

Fermi-LAT (Atwood et al. 2009) was unable to confirm the detection by AGILE, and an analysis ${ }^{1}$ of simultaneous data from the same direction yielded an upper limit (UL) of $10^{-7} \mathrm{ph} \mathrm{cm}^{-2} \mathrm{~s}^{-1}$ (95\% confidence level, CL) above $100 \mathrm{MeV}$, assuming a photon index $\Gamma=2$. A more extended analysis of Fermi-LAT data, including 3.5 years of data on the AGL $\mathrm{J} 2241+4454$ source location, did not lead to evidence of HE gamma-ray emission either. A $90 \%$ CL UL was set at the level of $9.4 \times 10^{-10} \mathrm{ph} \mathrm{cm}^{-2} \mathrm{~s}^{-1}$ for 3.5 years of observations (Mori et al. 2013).

The Be star MWC 656, also known as HD 215227, lies within the error bars of the AGILE best-fit source position. It was proposed as the optical counterpart of the excess claimed by the AGILE collaboration (Williams et al. 2010). The system displays optical photometric modulation with a period of $60.37 \pm$ 0.04 days (Williams et al. 2010; Paredes-Fortuny et al. 2012). Optical spectroscopic measurements of MWC 656 confirmed its binary nature (Casares et al. 2012). Recent optical spectroscopic measurements improved the spectral classification and reduced the uncertainties in the spectrophotometric distance, placing the system at a distance of $2.6 \pm 0.6 \mathrm{kpc}$. These measurements also revealed that the compact object is a stellar-mass $\mathrm{BH}$ of 3.86.9 solar masses, making this the first known case of a Be/BH system (Casares et al. 2014).

MWC 656 was also observed in radio with the European VLBI Network (EVN) and was not detected: Moldón (2012) reported $3 \sigma$ radio flux density ULs at $30-66 \mu \mathrm{Jy}$ level.

$\mathrm{X}$-ray observations were performed by XMM-Newton when the source was at an orbital phase $\phi=0.08^{2}$ (Munar-Adrover et al. 2014). The X-ray flux measured was compared with the radio ULs, resulting in a ratio compatible with the correlation derived in Corbel et al. (2013) for BH LMXBs, and similar to the faintest BH LMXBs detected. A search for hard X-ray emission has been conducted with INTEGRAL (Li et al. 2013) with no positive detection in the $18-60 \mathrm{keV}$ energy band reported for a total exposure time of $2.1 \mathrm{Ms}$.

In addition, the MAXI mission, which continouosly monitors the X-ray sky in the 2-20 keV band, has not detected emission coming from the AGL J2241+4454 position $^{3}$ on the same date as of the AGILE detection.

In this work we present the results of the observations of MWC 656 carried out with the MAGIC telescopes in 2012 and 2013. X-ray and optical observations were performed during the

\footnotetext{
1 http://fermisky.blogspot.com.es/2010/07/

extra-note-july-30-2010.html

2 Phase 0 has been set to the maximum of optical brightness, on HJD 2453243.3 (MJD 53 242.8). With the ephemeris from Casares et al. (2014), the periastron passage occurs at phase $0.01 \pm 0.10$.

3 http://maxi.riken.jp/top/index .php?cid=1\&jname= $\mathrm{J} 2242+447 \# 1 \mathrm{sp}$
}

Table 1. Observations of MWC 656 performed by MAGIC in 2012 and 2013.

\begin{tabular}{ccccc}
\hline \hline $\begin{array}{c}\text { Date } \\
(\mathrm{MJD})\end{array}$ & $\begin{array}{c}\text { Orbital } \\
\text { phase }\left(^{\circ}\right)\end{array}$ & $\begin{array}{c}\text { Zenith angle } \\
\text { range }\left(^{\circ}\right)\end{array}$ & $\begin{array}{c}\text { Time } \\
(\mathrm{h})\end{array}$ & Mode \\
\hline $56070-56078$ & $0.83-0.95$ & $23-50$ & 9.4 & mono \\
$56092-56097$ & $0.20-0.28$ & $22-51$ & 14.0 & mono \\
$56446-56448$ & $0.06-0.08$ & $28-45$ & 3.3 & stereo \\
\hline
\end{tabular}

2013 campaign to study the behavior of the source in a multiwavelength context.

\section{Observations}

The VHE observations of MWC 656 were carried out using the MAGIC telescopes, which are located at the observatory of El Roque de Los Muchachos $\left(28^{\circ} \mathrm{N}, 18^{\circ} \mathrm{W}, 2200 \mathrm{~m}\right.$ above sea level) on the island of La Palma, Canary Islands, Spain. The system consists of two $17 \mathrm{~m}$ diameter Imaging Atmospheric Cherenkov Telescopes (IACTs), each one with a pixelized camera containing photo-multipliers, covering a field of view of $3.5^{\circ}$. The current sensitivity of the MAGIC stereoscopic system is $0.71 \% \pm 0.02 \%$ of the Crab nebula flux in $50 \mathrm{~h}$ of observation for energies above $250 \mathrm{GeV}$ (MAGIC Collaboration et al. 2015). The spatial resolution at these energies is $\lesssim 0.1^{\circ}$ and the energy resolution is $\sim 18 \%$. In the case of monoscopic observations (also referred as mono observations) the integral sensitivity above $280 \mathrm{GeV}$ is about $1.6 \%$ of the Crab nebula flux in $50 \mathrm{~h}$ (Aliu et al. 2009). The observations are performed using wobble mode, in which the telescopes point at two different symmetric regions situated $0.4^{\circ}$ away from the source position to simultaneously evaluate the background.

The observations of MWC 656 were performed during two different epochs: May-June 2012, and June 2013. The observations in 2012 were performed between 23 May and 19 June in mono mode with MAGIC-II (the MAGIC-I telescope was not operational) for $23.4 \mathrm{~h}$. After selecting good-quality data, a total of $21.3 \mathrm{~h}$ remained. The 2013 observations were performed between 3 and 5 June, just after the periastron passage (see Fig. 1) in stereo mode. The source was observed for a total of $\sim 3.3 \mathrm{~h}$ during this period. A summary of the observations is shown in Table 1 .

The observation of 4 June, 2013 ( $\phi=0.08$ ) lasted for $\sim 1 \mathrm{~h}$ and was taken almost simultaneously with an XMM-Newton observation (XMM-Newton started observing immediately after MAGIC completed its observations), the results of which are reported in Munar-Adrover et al. (2014). MWC 656 was also observed with the fiber-fed STELLA Echelle Spectrograph (SES) of the $1.2 \mathrm{~m}$ robotic STELLA-I (ST) optical telescope (Strassmeier et al. 2004) at the Observatorio del Teide in Tenerife on the nights of 2, 3, 5, and 8 June 2013. The spectra cover the wavelength range 3870-8800 $\AA$ with increasing inter-order gaps starting at $7200 \AA$. The spectrograph provides an effective resolving power of $R=55000$. Two spectra were obtained on the nights of 2 and 5 June and one on the nights of 3 and 8 June. The integration time was set to $1800 \mathrm{~s}$ per spectrum, while the automatic pipeline products were used for the extraction and calibration of the spectra.

\section{Data analysis}

The MAGIC data analysis was performed using the standard MAGIC analysis and reconstruction software, MARS 


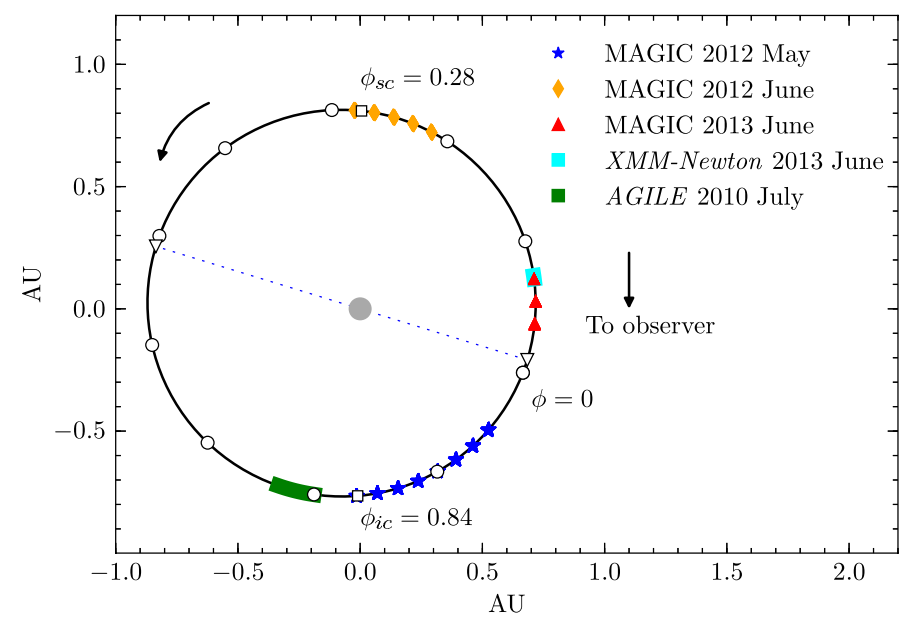

Fig. 1. Depiction of the orbit of the MWC 656 system as seen from above. The optical star MWC 656 lies at the focus of the ellipse, and the $\mathrm{BH}$ follows the elliptical orbit. The size of the star is scaled with respect to the BH orbit. The MAGIC, XMM-Newton, and AGILE observations are overlaid along the orbit. Circles represent steps of 0.1 orbital phases, while triangles mark the periastron and apastron phases, which are linked by a dotted line. Squares mark the position of the inferior and superior conjunctions.

(Zanin et al. 2013). The recorded shower images were calibrated, cleaned, and parametrized (Hillas 1985; Aliu et al. 2009). The $\gamma /$ hadron separation (background rejection) was performed with the random forest (RF) method (Albert et al. 2008). The event direction and energy of the primary gamma ray were also reconstructed by using an RF method for the mono observations. The energy of each event for stereoscopic observations was estimated using look-up tables generated by Monte Carlo simulations (Aleksić et al. 2012). Upper limits (ULs) were derived using the method explained in Rolke et al. (2005) with a CL of $95 \%$ and a systematic uncertainty of $30 \%$, assuming different photon indexes ( $\Gamma=2.0,2.5$, and 3.0). The values obtained for the three spectral indexes are compatible at the 5\% level. The results reported in this paper are for $\Gamma=2.5$.

\section{Results}

No significant gamma-ray emission was detected from MWC 656 in either observational campaign. Furthermore, no significant signal was detected in a day-to-day analysis.

We computed $95 \%$ confidence level (CL) integral flux ULs above $300 \mathrm{GeV}$. The integral flux UL for the whole observational campaign of MWC 656 is $F(E>300 \mathrm{GeV})=2.0 \times$ $10^{-12} \mathrm{~cm}^{-2} \mathrm{~s}^{-1}(\sim 2.4 \%$ of the Crab nebula flux at the same energy and corresponding to a luminosity $L_{\mathrm{VHE}} \sim 10^{33} \mathrm{erg} \mathrm{s}^{-1}$ ) at $95 \% \mathrm{CL}$, assuming a power-law model with a photon index $\Gamma=2.5$.

We divided the observational periods into four different phase bins, using a bin width of 0.1 in phase, along with the most recent ephemeris (Casares et al. 2014). The phase was binned as follows: phases $0.8-0.9,0.9-1.0$, and $0.2-0.3$ for the 2012 campaign, covering the orbit before the periastron passage and also post-periastron phases (see Fig. 1). The 2013 campaign covered the phase range $0.0-0.1$, just after the periastron passage. No signal was detected, and integral ULs for bins of $\sim 0.1$ in phase were computed as well (see Table 2).
Table 2. Integral flux ULs for $E>300 \mathrm{GeV}$ calculated at 95\% CL for MWC 656 for each orbital phase range.

\begin{tabular}{ccccc}
\hline \hline Mode & Phase bin & $\begin{array}{c}\text { Integral UL } \\
(E>300 \mathrm{GeV}) \\
{\left[10^{-12} \mathrm{~cm}^{-2} \mathrm{~s}^{-1}\right]}\end{array}$ & Significance & $t_{\text {eff }}$ \\
\hline Stereo & $0.0-0.1$ & 2.0 & 1.0 & {$[\mathrm{~h}]$} \\
Mono & $0.2-0.3$ & 8.7 & 2.1 & 3.3 \\
Mono & $0.8-0.9$ & 6.5 & 1.0 & 11.5 \\
Mono & $0.9-1.0$ & 2.5 & -1.1 & 4.9 \\
\hline
\end{tabular}

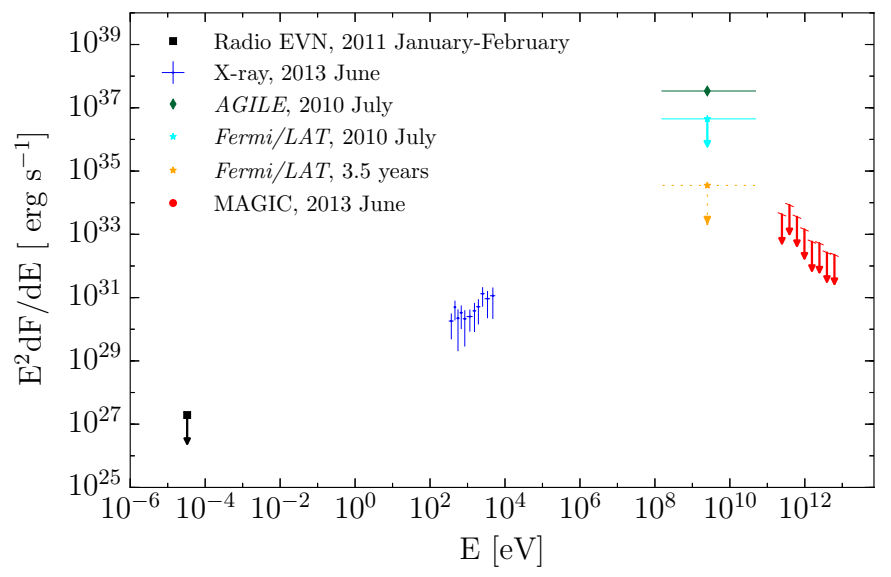

Fig. 2. SED of MWC 656 including MAGIC ULs from the 2013 campaign together with simultaneous XMM-Newton data from Munar-Adrover et al. (2014). We also include EVN radio ULs from Moldón (2012), the AGILE energy flux from Lucarelli et al. (2010) and the Fermi-LAT UL simultaneous (green) to the AGILE measurement. The 3.5-year UL set by Fermi-LAT to any persistent emission is also plotted.

We computed differential flux ULs from the energy threshold of our analysis $(245 \mathrm{GeV})$ up to $6.3 \mathrm{TeV}$ at $95 \% \mathrm{CL}$, with five bins per decade of energy (see Fig. 2).

The MAGIC observations carried out on 4 June, 2013 were performed almost simultaneously with an XMM-Newton observation. The detected low X-ray flux was consistent with the source being in the quiescent state (defined in terms of the Eddington luminosity, when $L<10^{-5} L_{\text {Edd }}$ ) during the observation (Munar-Adrover et al. 2014). The MAGIC integral flux $\mathrm{UL}$ for 4 June is $F(E>300 \mathrm{GeV})<4.9 \times 10^{-12} \mathrm{~cm}^{-2} \mathrm{~s}^{-1}$. There is no specific information about the $\mathrm{X}$-ray state of the binary system during the 2012 observations. Other space missions such as MAXI have not reported emission from MWC 656 during the 2012-2013 campaign, which might be indicative of a quiescent state as well.

Finally, the STELLA spectra, contemporaneous with the 2013 MAGIC campaign, show the double peaked He II $\lambda 4686$ emission line with an equivalent width similar to that reported in Casares et al. (2014). We also detect other emission lines, mainly $\mathrm{H} \alpha, \mathrm{H} \beta$, and weak FeII lines with a similar strength to that measured by Casares et al. (2012). Therefore, we conclude that MWC 656 is in a similar optical state as in past observations, the 2013 X-ray observations indicate a quiescent state, and hence the accretion activity should be very similar.

\section{Discussion and conclusions}

We have searched for a VHE counterpart of the only known $\mathrm{Be} / \mathrm{BH}$ binary system, MWC 656. The VHE observations 
performed by MAGIC can exclude a VHE flux based on the extrapolation of the emission from the AGILE detection. Assuming a power-law spectrum and a photon index $\Gamma=2.5$, this emission would be $\sim 4 \times 10^{-11} \mathrm{TeV}^{-1} \mathrm{~cm}^{-2} \mathrm{~s}^{-1}$ at $300 \mathrm{GeV}$ $\left(L_{\mathrm{VHE}} \sim 2 \times 10^{34} \mathrm{erg} \mathrm{s}^{-1}\right)$, which is well above the UL imposed by MAGIC. However, no flaring episodes were reported during the MAGIC observations, limiting the conclusions we can derive from the HE/VHE comparison.

In this type of binary, several mechanisms have been proposed that would result in gamma-ray emission above tens of GeV (Remillard \& McClintock 2006; Fender 2006; Zdziarski et al. 2014). Unfortunately, the lack of contemporaneous data at other wavelengths during the AGILE flare make conclusions on the type of emission model highly speculative. It is even possible that the AGILE detection was just a transient event of an unknown nature in the direction of the binary system but not related to it. Nevertheless, different emission levels could be expected depending on the state of the system, that is, quiescence or accreting.

During simultaneous X-ray and VHE observations the X-ray luminosity of the source in the $0.3-5.5 \mathrm{keV}$ energy range was $L_{\mathrm{X}}(0.3-5.5 \mathrm{keV})=\left(1.6_{-0.9}^{+1.0}\right) \times 10^{31} \mathrm{erg} \mathrm{s}^{-1} \equiv(3.1 \pm$ $2.3) \times 10^{-8} L_{\text {Edd }}$ (Munar-Adrover et al. 2014) for the estimated BH mass range 3.8-6.9 $M_{\odot}$ (Casares et al. 2014). The low $\mathrm{X}$-ray luminosity is characteristic of systems in quiescent states (defined in terms of the Eddington luminosity, when $L_{X}<$ $10^{-5} L_{\text {Edd }}$, Plotkin et al. 2013). For instance, the X-ray luminosity is $\sim 5$ orders of magnitude lower than the one typically observed in Cygnus X-1, which has also been observed by MAGIC (Albert et al. 2007). Even if we consider an increase in the X-ray flux consistent with a flaring state in the 2012 observations and a ratio between X-rays and VHE gamma rays of $F_{\mathrm{X}} / F_{\mathrm{TeV}} \sim 10$ (similar to that observed for Cygnus X-1 in Albert et al. 2007), the expected VHE emission would be a factor $\sim 1.5 \times 10^{-5}$ of the Crab nebula flux $\left(\sim 2 \times 10^{-15} \mathrm{~cm}^{-2} \mathrm{~s}^{-1}\right)$ in this case, also well below the detectable levels for the current IACTs and even for the next generation of Cherenkov telescopes: the Cherenkov Telescope Array (CTA). The integral sensitivity of CTA is predicted to reach $\sim 3 \times 10^{-13} \mathrm{TeV} \mathrm{cm}^{-2} \mathrm{~s}^{-1}$ above $50 \mathrm{GeV}$ for $50 \mathrm{~h}$ of observation $\left(\sim 7 \times 10^{-12} \mathrm{TeV} \mathrm{cm}^{-2} \mathrm{~s}^{-1}\right.$ in $1 \mathrm{~h}$ observation, considering array E configuration; Acharya et al. 2013), still not enough to detect MWC 656 in relatively short time in this simple approximation.

Figure 2 shows the spectral energy distribution (SED) of MWC 656. The absence of a detection of steady emission at high and very high energies implies that the X-ray emission cannot continue to increase with energy indefinitely and must turn over in the SED. The MAGIC differential ULs correspond only to data of June 2013 because for the 2012 observations X-ray information is not available. We also plotted the AGILE measurement in the SED along with the Fermi-LAT upper limit, obtained with observations performed on the same dates of the AGILE detection. Although the Fermi-LAT UL contradicts the AGILE detection, it is worth noting that the observation modes of these telescopes are different and that the integration time might not be exactly the same. Therefore, the observations are not strictly simultaneous and Fermi-LAT might have missed short $(<1 \mathrm{~h})$ gamma-ray flares from MWC 656.

Acknowledgements. We would like to thank the Instituto de Astrofísica de Canarias for the excellent working conditions at the Observatorio del Roque de los Muchachos in La Palma. The support of the German BMBF and MPG, the Italian INFN, the Swiss National Fund SNF, and the ERDF funds under the Spanish MINECO is gratefully acknowledged. This work was also supported by the CPAN CSD2007-00042 and MultiDark CSD2009-00064 projects of the Spanish Consolider-Ingenio 2010 programme, by grant 127740 of the Academy of Finland, by the Croatian Science Foundation (HrZZ) Project 09/176, by the DFG Collaborative Research Centers SFB823/C4 and SFB876/C3, and by the Polish MNiSzW grant 745/N-HESS-MAGIC/2010/0. J.C. acknowledges support by the Spanish Ministerio de Economía y Competividad (MINECO) under grant AYA2010-18080. The authors thank the anonymous referee for a thorough review and a very constructive list of remarks that helped improving the quality and clarity of this manuscript.

\section{References}

Acharya, B. S., Actis, M., Aghajani, T., et al. 2013, Astropart. Phys., 43, 3 Albert, J., Aliu, E., Anderhub, H., et al. 2007, ApJ, 665, L51

Albert, J., Aliu, E., Anderhub, H., et al. 2008, Nucl. Instrum. Methods, 588, 424 Aleksić, J., Alvarez, E. A., Antonelli, L. A., et al. 2012, Astropart. Phys., 35, 435 Aliu, E., Anderhub, H., Antonelli, L. A., et al. 2009, Astropart. Phys., 30, 293 Atwood, W. B., Abdo, A. A., Ackermann, M., et al. 2009, ApJ, 697, 1071 Bodaghee, A., Tomsick, J. A., Pottschmidt, K., et al. 2013, ApJ, 775, 98

Casares, J., Ribó, M., Ribas, I., et al. 2012, MNRAS, 421, 1103

Casares, J., Negueruela, I., Ribó, M., et al. 2014, Nature, 505, 378

Corbel, S., Coriat, M., Brocksopp, C., et al. 2013, MNRAS, 428, 2500

Dubus, G. 2013, A\&ARv, 21, 64

Fender, R. 2006, in Relativistic Jets: The Common Physics of AGN, Microquasars, and Gamma-Ray Bursts, eds. P. A. Hughes, \& J. N. Bregman, AIP Conf. Ser., 856, 23

Fermi-LAT Collaboration, Abdo, A. A., Ackermann, M., Ajello, M., et al. 2009, Science, 326, 1512

Hillas, A. M. 1985, Internat. Cosm. Ray Conf., 3, 445

Li, J., Torres, D. F., Zhang, S., \& Wang, J. 2013 [arXiv: 1302. 5211]

Lucarelli, F., Verrecchia, F., Striani, E., et al. 2010, The Astronomer's Telegram, 2761, 1

MAGIC Collaboration, Aleksic, J., Ansoldi, S., et al. 2015, Astropart. Phys., in press [arXiv: 1409.5594]

Malyshev, D., Zdziarski, A. A., \& Chernyakova, M. 2013, MNRAS, 434, 2380

Moldón, J. 2012, Ph.D. Thesis, Universitat de Barcelona

Mori, M., Kawachi, A., Nagataki, S., \& Naito, T. 2013, 2012 Fermi Symp. Proc. eConf C121028

Munar-Adrover, P., Paredes, J. M., Ribó, M., et al. 2014, ApJ, 786, L11

Paredes-Fortuny, X., Ribó, M., Fors, O., \& Núñez, J. 2012, in AIP Conf. Ser. 1505, eds. F. A. Aharonian, W. Hofmann, \& F. M. Rieger, 390

Plotkin, R. M., Gallo, E., \& Jonker, P. G. 2013, ApJ, 773, 59

Remillard, R. A. \& McClintock, J. E. 2006, ARA\&A, 44, 49

Rolke, W. A., López, A. M., \& Conrad, J. 2005, Nucl. Instrum. Methods, 551, 493

Sabatini, S., Tavani, M., Coppi, P., et al. 2013, ApJ, 766, 83

Strassmeier, K. G., Granzer, T., Weber, M., et al. 2004, Astron. Nachr., 325, 527

Tavani, M., Barbiellini, G., Argan, A., et al. 2009a, A\&A, 502, 995

Tavani, M., Bulgarelli, A., Piano, G., et al. 2009b, Nature, 462, 620

Williams, S. J., Gies, D. R., Matson, R. A., et al. 2010, ApJ, 723, L93

Zanin, R., Carmona, E., \& Sitarek, J. 2013, in Proc. of the ICRC 2013, International Cosmic Ray Conference

Zdziarski, A. A., Stawarz, Ł., Pjanka, P., \& Sikora, M. 2014, MNRAS, 440, 2238

1 IFAE, Campus UAB, 08193 Bellaterra, Spain

2 Università di Udine, and INFN Trieste, 33100 Udine, Italy

3 INAF National Institute for Astrophysics, 00136 Rome, Italy

4 Università di Siena, and INFN Pisa, 53100 Siena, Italy

5 Croatian MAGIC Consortium, Rudjer Boskovic Institute, University of Rijeka and University of Split, 10000 Zagreb, Croatia

6 Max-Planck-Institut für Physik, 80805 München, Germany

7 Universidad Complutense, 28040 Madrid, Spain

8 Inst. de Astrofísica de Canarias, 38200 La Laguna, Tenerife, Spain

9 University of Łódź, 90236 Lodz, Poland

10 Deutsches Elektronen-Synchrotron (DESY), 15738 Zeuthen, Germany

11 ETH Zurich, 8093 Zurich, Switzerland

12 Universität Würzburg, 97074 Würzburg, Germany

13 Centro de Investigaciones Energéticas, Medioambientales y Tecnológicas, 28040 Madrid, Spain

14 Institute of Space Sciences, 08193 Barcelona, Spain

15 Università di Padova and INFN, 35131 Padova, Italy

16 Technische Universität Dortmund, 44221 Dortmund, Germany 
17 Unitat de Física de les Radiacions, Departament de Física, and CERES-IEEC, Universitat Autònoma de Barcelona, 08193 Bellaterra, Spain

18 Universitat de Barcelona, ICC, IEEC-UB, 08028 Barcelona, Spain

19 Japanese MAGIC Consortium, Division of Physics and Astronomy, Kyoto University, Japan

20 Finnish MAGIC Consortium, Tuorla Observatory, University of Turku and Department of Physics, University of Oulu, 90014 Oulun, Finland

21 Inst. for Nucl. Research and Nucl. Energy, 1784 Sofia, Bulgaria

22 Università di Pisa, and INFN Pisa, 56126 Pisa, Italy

23 ICREA and Institute of Space Sciences, 08193 Barcelona, Spain

24 Università dell'Insubria and INFN Milano Bicocca, Como, 22100 Como, Italy
25 Now at: NASA Goddard Space Flight Center, Greenbelt, MD 20771, USA and Department of Physics and Department of Astronomy, University of Maryland, College Park, MD 20742, USA

26 Now at: École polytechnique fédérale de Lausanne (EPFL), 1015 Lausanne, Switzerland

27 Now at: Institut für Astro- und Teilchenphysik, Leopold-FranzensUniversität Innsbruck, 6020 Innsbruck, Austria

${ }^{28}$ Now at: Finnish Centre for Astronomy with ESO (FINCA), 20014 Turku, Finland

29 Now at: Astrophysics Science Division, Bhabha Atomic Research Centre, 400085 Mumbai, India

30 Also at INAF-Trieste

31 Now at: ASTRON Netherlands Institute for Radio Astronomy, Oude Hoogeveensedijk 4, 7991 PD Dwingeloo, The Netherlands 